\title{
Monocentric Retrospective Study Assessing the Association Between Clinical and Subclinical Hypothyroidism and Type 2 Diabetes Mellitus (Type 2 DM) and the Benefit of Treating Hypothyroidism on the Improvement of Diabetes Control
}

\section{Mohammad Rida Farhat ${ }^{1 *}$, Khaled Soukarieh ${ }^{2}$, Bilal Jamal El Din ${ }^{2}$ and Batoul Mcheik ${ }^{3}$}

${ }^{1}$ Department of General Surgery, Faculty of Medical Sciences, Lebanese University, Beirut, Lebanon

${ }^{2}$ Department of Internal Medicine, Faculty of Medical Sciences, Lebanese University, Beirut, Lebanon

${ }^{3}$ General Medicine Intern, Faculty of Medical Sciences, Lebanese University, Beirut, Lebanon

*Corresponding Author: Mohammad Rida Farhat, Department of General Surgery, Faculty of Medical Sciences, Lebanese University, Beirut, Lebanon.
Received: February 24, 2021

Published: April 14, 2021

(C) All rights are reserved by Mohammad Rida

Farhat, et al.

\begin{abstract}
Background: Diabetes prevalence was as high as 14.6\% in Lebanon in 2017. A lot of studies suggest an association between clinical and subclinical hypothyroidism, and type $2 \mathrm{DM}$, but with small sample sizes.

Aim: This study aimed to determine the prevalence of hypothyroidism among Lebanese diabetic patients, to assess the benefit of treating hypothyroidism on the improvement of diabetes, and to check whether a screening of hypothyroidism could be beneficial in all diabetic patients.

Methods: A monocentric cross sectional study was conducted to randomly recruit 100 patients having two HbA1C and two TSH readings, among diabetic patients. Data was collected from 2010 till 2017. The prevalence of hypothyroidism was $11.99 \%$ among Lebanese diabetic patients.

Results: Mean HbA1C level before hypothyroidism treatment was 8.84 and decreased to 8.26 afterwards. This study showed that the patients who received hypothyroidism treatment had significantly lower HbA1C levels (p-value $=0.001$ ). However, statistically significant $\mathrm{HbA1C}$ reduction was shown in women ( $\mathrm{p}$-value $<0.001)$, and in patients who didn't have any comorbidities $(\mathrm{p}$-value $=0.005)$. Conclusion: The prevalence of hypothyroidism among diabetic patients was determined and proved the utility of treating it to improve $\mathrm{HbA1C}$ levels.
\end{abstract}

Keywords: Diabetes; Hypothyroidism; HbA1C

\section{Introduction}

Diabetes mellitus is by far the most common chronic endocrine disease nowadays. Its main characteristic is an impaired insulin secretion and/or insulin action that results in a hyperglycemia [1]. By 2033, diabetes mellitus will affect about 600 million people worldwide. Its global prevalence will be around $11.1 \%$ by then [2]. In Lebanon, $14.6 \%$ of Lebanese adults were estimated to have diabetes in 2017. This includes about 585,500 patients [3].

There is a lot of evidence suggesting that thyroid disorders (specifically clinical and subclinical hypothyroidism) and type 2 
Monocentric Retrospective Study Assessing the Association Between Clinical and Subclinical Hypothyroidism and Type 2 Diabetes Mellitus (Type 2 DM) and the Benefit of Treating Hypothyroidism on the Improvement of Diabetes Control

DM are associated. However, these studies have small sample sizes and their data is inconclusive in its majority. That's why this topic seems to be an excellent target for future studies [4]. While a higher prevalence of thyroid disorders (13.4\%) was observed in type 2 diabetic patients than the general population, some gender differences were observed: a higher prevalence of $31.4 \%$ was noted in female type 2 DM patients, compared to $6.9 \%$ in male patients [5].

The most probable mechanism leading to type2 DM in thyroid dysfunction could be attributed to physiological aberrations that lead to impaired glucose utilization and disposal in muscles. In hypothyroidism, there is a reduction in glucose-induced insulin secretion by beta cells caused by perturbed expression of glucose transporter type 2 gene. Moreover, due to reduced renal clearance of insulin in hypothyroid conditions, physiological requirements of insulin were diminished. Also, insulin resistance has been shown to be caused in hypothyroidism in various in vitro and preclinical studies where it was found that peripheral muscles became less responsive in hypothyroid conditions [6].

Moreover, subclinical hypothyroidism was also associated with renal injury in diabetic patients [7], with chronic kidney disease [8], and diabetic nephropathy [9]. A higher prevalence of cerebrovascular diseases was noted among type 2 diabetes mellitus patients with hypothyroidism compared to those with euthyroidism after adjustment for age and gender [10].

Furthermore, treating hypothyroidism had lots of advantages. Thyroxine replacement therapy has protective effects on the renal function and the cardiovascular system as well [11]. That's why evidence supports the necessity to screen thyroid function in patients with type 2 diabetes $[12,13]$ and appropriate treatments should be given accordingly as well [4].

Hence, our article will focus on hypothyroidism in diabetic patients and the impact of hypothyroidism treatment on diabetes control. Therefore, a retrospective cross sectional study, carried on in Mount Lebanon Hospital was conducted between $1^{\text {st }}$ January 2010 and $1^{\text {st }}$ January 2018.

\section{Methods}

Study design

It is a retrospective cross sectional study, carried on during 2018 in Mount Lebanon Hospital.

No written consent was necessary from the patients included in our study, nor from their physicians, because it was leaded using the medical records from the hospitals' archives.

\section{Study population}

The study population consisted of all diabetic patients who presented to Mount Lebanon Hospital between December 2010 and June 2017. The sample was collected from the diabetic patients who took TSH tests.

\section{Inclusion criteria}

All patients having two $\mathrm{HbA1C}$ readings at Mount Lebanon hospital, as well as two TSH level were included in our study.

\section{Exclusion criteria}

We exclude Patients who didn't follow up in Mount Lebanon Hospital, who didn't have both TSH and HbA1C tests results in the medical record.

\section{Data collection}

Data was collected from the achieved medical records at the Hospital for the period between December 2010 and June 2017 using a uniform data collection form taking into consideration Sociodemographic data: age, and gender, Comorbidities, BMI, Diabetes diagnosis date, Diabetes treatment, HbA1C before and after hypothyroidism treatment, TSH levels (before and after treatment).

\section{Statistical analysis}

We used Excel for data entry and for the tables and graphs used as study results.

A descriptive analysis was first done to assess age, gender, comorbidities, BMI, diabetes duration, HbA1C before and after hypothyroidism treatment, FT4 levels, as well as TSH before and after treatment. Then, the differences between treated and untreated hypothyroidism were assessed using Related-Samples Wilcoxon Signed Rank Test, since the variables weren't normally distributed, and each patient had two measures of the TSH and HbA1C. The significance level of the test was admitted as p-value $<0.05$.

For the prevalence calculation, we used the formula: Prevalence $=($ Number of cases in a given period $* 100000) /$ Total population during the same period [14]. Because the population considered in this stud was the patients admitted to one center, Mount Lebanon Hospital, then the prevalence calculated is a specific prevalence.

\section{Results}

Prevalence of hypothyroidism

There were in total 13874 diabetic patients admitted to Mount Lebanon Hospital during the period between December 2010 and June 2017. Among these, 1664 patients were hypothyroid. Prevalence of hypothyroidism was then $11.9936572 \%$. 
Monocentric Retrospective Study Assessing the Association Between Clinical and Subclinical Hypothyroidism and Type 2 Diabetes Mellitus (Type 2 DM) and the Benefit of Treating Hypothyroidism on the Improvement of Diabetes Control

\section{Descriptive statistics}

Patients had diabetes since 14 years in mean. Results show that the mean patients' age was 69 years, mean BMI 26.95, mean hemoglobin 11.98. As for TSH, it was 12.97 before treatment, and 8.27 afterwards. HbA1C before hypothyroidism treatment was 8.84 , and 8.26 afterwards (Table 1 ).

\begin{tabular}{|l|c|c|c|c|}
\hline & Median & Std. & Minimum & Maximum \\
\cline { 3 - 5 } & Deviation & & \\
\hline BMI & 26 & 4.16759 & 19 & 39 \\
\hline Age & 71 & 11.901 & 42 & 93 \\
\hline $\begin{array}{l}\text { FT4 upon } \\
\text { hypothyroidism } \\
\text { diagnosis }\end{array}$ & 0.4 & 0.31668 & 0.02 & 1.3 \\
\hline $\begin{array}{l}\text { TSH before } \\
\text { treatment }\end{array}$ & 7.9 & 13.98471 & 5.02 & 91 \\
\hline TSH after treatment & 6.7 & 7.48416 & 0.4 & 40.3 \\
\hline $\begin{array}{l}\text { Diabetes duration } \\
\text { HBA1C before } \\
\text { hypothyroidism } \\
\text { treatment }\end{array}$ & 12 & 6.99758 & 3 & 36 \\
\hline $\begin{array}{l}\text { HBA1C after } \\
\text { treatment }\end{array}$ & 7.8 & 1.80342 & 6.2 & 16 \\
\hline $\begin{array}{l}\text { Hemoglobin } \\
\text { Number } \\
\text { comorbidities }\end{array}$ & 12 & 2.00807 & 7.9 & 17 \\
\hline
\end{tabular}

Table 1: Descriptive statistics.

Half of the patients were treated for hypothyroidism whereas the others didn't receive any hypothyroidism treatment (Figure 1).

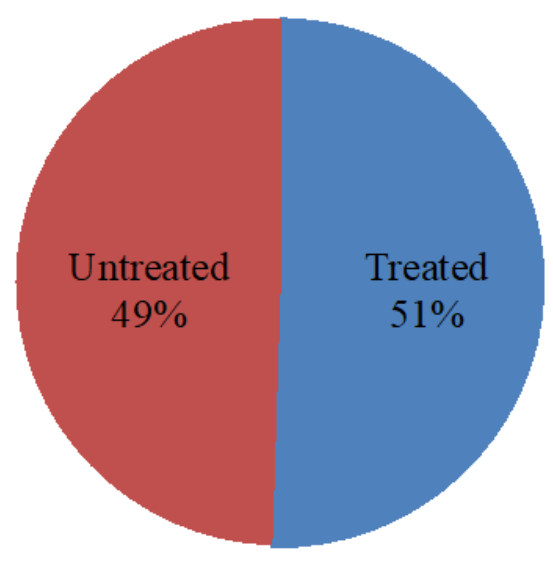

Figure 1: Hypothyroidism treatment status.
Figure 2 shows the number of comorbidities per patient. Almost half the patients (45\%) had one comorbidity (alongside with diabetes and thyroid dysfunction), whereas the quarter (26\%) didn't have any comorbidity.

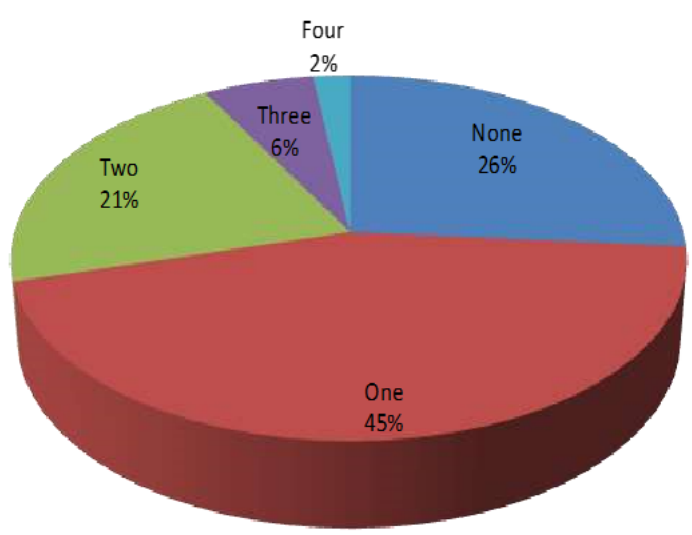

Figure 2: Number of comorbidities per patient.

Figure 3 shows that the majority of the patients' comorbidities were hypertension (37 patients) and dyslipidemia (23 patients).

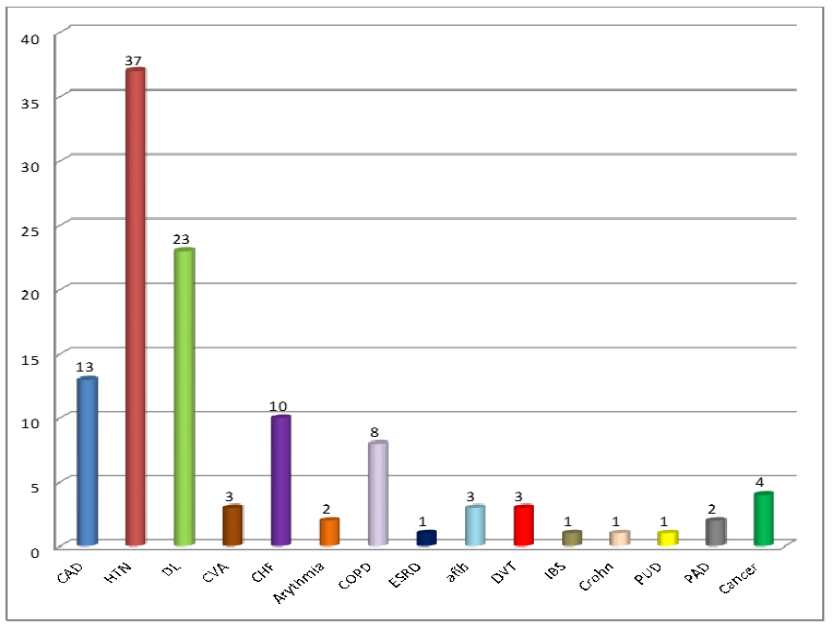

Figure 3: Comorbidities: CAD: Coronary Artery Disease; HTN: Hypertension; DL: Dyslipidemia; CVA: Cerebrovascular Accident; CHF: Congestive Heart Failure; COPD: Chronic Obstructive Pulmonary Disease; ESRD: End Stage Renal Disease; afib: Atrial Fibrillation; DVT: Deep Venous Thrombosis; IBS: Irritable Bowel Syndrome; PUD: Peptic Ulcer Disease; PAD: Peripheral Arterial Disease. 
Monocentric Retrospective Study Assessing the Association Between Clinical and Subclinical Hypothyroidism and Type 2 Diabetes Mellitus (Type 2 DM) and the Benefit of Treating Hypothyroidism on the Improvement of Diabetes Control

Hypothyroidism treatment and diabetes control

The median TSH and median HbA1C are significantly lower in the group that received hypothyroidism treatment (Table 2).
Although both groups had significantly lower TSH results after hypothyroidism treatment, a statistically significant reduction of median HbA1C was only observed in women (Table 3).

\begin{tabular}{|c|c|c|c|c|}
\hline & Null Hypothesis & Test & $P$ value & Decision \\
\hline \multirow{6}{*}{$\begin{array}{l}\text { Untreated } \\
\text { hypothyroidism }\end{array}$} & \multirow{3}{*}{$\begin{array}{l}\text { The median of differences } \\
\text { between the two TSH results } \\
\text { readings equals } 0 .\end{array}$} & Related-Samples & \multirow{3}{*}{0.46} & \multirow{3}{*}{$\begin{array}{c}\text { Retain the null } \\
\text { hypothesis. }\end{array}$} \\
\hline & & Wilcoxon Signed & & \\
\hline & & Rank Test & & \\
\hline & \multirow{3}{*}{$\begin{array}{c}\text { The median of differences } \\
\text { between the two HBA1C results } \\
\text { readings equals } 0 .\end{array}$} & Related-Samples & \multirow{3}{*}{0.091} & \multirow{3}{*}{$\begin{array}{c}\text { Retain the null } \\
\text { hypothesis. }\end{array}$} \\
\hline & & Wilcoxon Signed & & \\
\hline & & Rank Test & & \\
\hline \multirow{6}{*}{$\begin{array}{l}\text { Treated } \\
\text { hypothyroidism }\end{array}$} & \multirow{3}{*}{$\begin{array}{l}\text { The median of differences } \\
\text { between the two TSH results } \\
\text { readings equals } 0 .\end{array}$} & Related-Samples & \multirow{3}{*}{$<0.001$} & \multirow{3}{*}{$\begin{array}{c}\text { Reject the null } \\
\text { hypothesis. }\end{array}$} \\
\hline & & Wilcoxon Signed & & \\
\hline & & Rank Test & & \\
\hline & \multirow{3}{*}{$\begin{array}{c}\text { The median of differences } \\
\text { between the two HBA1C results } \\
\text { readings equals } 0 .\end{array}$} & Related-Samples & \multirow{3}{*}{0.001} & \multirow{3}{*}{$\begin{array}{l}\text { Reject the null } \\
\text { hypothesis. }\end{array}$} \\
\hline & & Wilcoxon Signed & & \\
\hline & & Rank Test & & \\
\hline
\end{tabular}

Table 2: Hypothyroidism treatment and diabetes control.

\begin{tabular}{|c|c|c|c|c|}
\hline & Null Hypothesis & Test & Sig. & Decision \\
\hline \multirow[t]{6}{*}{ Female } & \multirow{3}{*}{$\begin{array}{c}\text { The median of differences between the two TSH } \\
\text { results readings equals } 0 .\end{array}$} & Related-Samples & \multirow[t]{3}{*}{0.013} & \multirow[t]{3}{*}{ Reject the null hypothesis. } \\
\hline & & Wilcoxon Signed & & \\
\hline & & Rank Test & & \\
\hline & \multirow{3}{*}{$\begin{array}{l}\text { The median of differences between the two HBA1C } \\
\text { results readings equals } 0 .\end{array}$} & Related-Samples & \multirow[t]{3}{*}{$<0.001$} & \multirow[t]{3}{*}{ Reject the null hypothesis } \\
\hline & & Wilcoxon Signed & & \\
\hline & & Rank Test & & \\
\hline \multirow[t]{6}{*}{ Male } & \multirow{3}{*}{$\begin{array}{c}\text { The median of differences between the two TSH } \\
\text { results readings equals } 0 .\end{array}$} & Related-Samples & \multirow[t]{3}{*}{0.001} & \multirow[t]{3}{*}{ Reject the null hypothesis. } \\
\hline & & Wilcoxon Signed & & \\
\hline & & Rank Test & & \\
\hline & \multirow{3}{*}{$\begin{array}{c}\text { The median of differences between the two HBA1C } \\
\text { results readings equals } 0 .\end{array}$} & Related-Samples & \multirow[t]{3}{*}{0.108} & \multirow[t]{3}{*}{ Retain the null hypothesis } \\
\hline & & Wilcoxon Signed & & \\
\hline & & Rank Test & & \\
\hline \multicolumn{4}{|c|}{ Asymptotic significances are displayed. The significance level is 0.05 . } & \\
\hline
\end{tabular}

Table 3: Gender and diabetes control.

Table 4 shows that in patients who had no comorbidities, HbA1C median was significantly lower after hypothyroidism treatment, compared to the patients who had one or more comorbidities.

\section{Discussion}

We conducted a retrospective cross sectional study, carried on during the period between December 2010 and June 2017, to 
Monocentric Retrospective Study Assessing the Association Between Clinical and Subclinical Hypothyroidism and Type 2 Diabetes Mellitus (Type 2 DM) and the Benefit of Treating Hypothyroidism on the Improvement of Diabetes Control

\begin{tabular}{|c|c|c|c|c|}
\hline & Null Hypothesis & Test & Sig. & Decision \\
\hline \multirow{3}{*}{ None } & \multirow{3}{*}{$\begin{array}{l}\text { The median of differences between the } \\
\text { two HBA1C results readings equals } 0 \text {. }\end{array}$} & Related-Samples & \multirow{3}{*}{0.005} & \multirow{3}{*}{ Reject the null hypothesis. } \\
\hline & & Wilcoxon Signed & & \\
\hline & & Rank Test & & \\
\hline \multirow{3}{*}{ One } & \multirow{3}{*}{$\begin{array}{l}\text { The median of differences between the } \\
\text { two HBA1C results readings equals } 0 \text {. }\end{array}$} & Related-Samples & \multirow{3}{*}{0.073} & \multirow{3}{*}{ Retain the null hypothesis. } \\
\hline & & Wilcoxon Signed & & \\
\hline & & Rank Test & & \\
\hline \multirow{3}{*}{ Two } & \multirow{3}{*}{$\begin{array}{l}\text { The median of differences between the } \\
\text { two HBA1C results readings equals } 0 \text {. }\end{array}$} & Related-Samples & \multirow{3}{*}{0.099} & \multirow{3}{*}{ Retain the null hypothesis } \\
\hline & & Wilcoxon Signed & & \\
\hline & & Rank Test & & \\
\hline \multirow{3}{*}{ Three } & \multirow{3}{*}{$\begin{array}{l}\text { The median of differences between the } \\
\text { two HBA1C results readings equals } 0 \text {. }\end{array}$} & Related-Samples & \multirow{3}{*}{0.753} & \multirow{3}{*}{ Retain the null hypothesis. } \\
\hline & & Wilcoxon Signed & & \\
\hline & & Rank Test & & \\
\hline \multirow{3}{*}{ Four } & \multirow{3}{*}{$\begin{array}{l}\text { The median of differences between the } \\
\text { two HBA1C results readings equals } 0 \text {. }\end{array}$} & Related-Samples & \multirow{3}{*}{0.18} & \multirow{3}{*}{ Retain the null hypothesis. } \\
\hline & & Wilcoxon Signed & & \\
\hline & & Rank Test & & \\
\hline
\end{tabular}

Table 4: Number of comorbidities and diabetes control.

determine the prevalence rate of hypothyroidism, among type 2 diabetic patients who were hospitalized in Mount Lebanon Hospital during that period, and to prove the benefit of treating hypothyroidism on type 2 diabetes control, as well as the necessity to screen all diabetic patients for hypothyroidism eventually.

The hypothyroidism prevalence was $11.99 \%$ in our study. This is close to the prevalence of $13.4 \%$ reported in diabetic population [6]. However, in another study, thyroid disorders prevalence was $48 \%$ in type 2 diabetic patients [15], much higher than ours. This finding suggests the need for a study with a larger and more representative sample of the Lebanese population.

In our study, patients had diabetes duration of 14 years in mean. This finding is supported by the fact that having diabetes for longer than ten years was a risk factor significantly associated with thyroid dysfunction [5]. Furthermore, metformin had a TSH lowering effect that was not dependent on long term metformin therapy [16]. Consequently, having diabetes for a longer duration and being treated for it higher the risk of having thyroid dysfunction. This could explain why our patients had a long duration of diabetes.

Our study showed that the patients who received hypothyroidism treatment (showing also a significant reduction of TSH levels after treatment) had significantly lower HbA1C levels. This joins the findings of a cross sectional study including 7966 patients with type 2 diabetes mellitus, that suggested an association between poor glycemic control and the risk of subclinical hypothyroidism [17]. In fact, abundant evidence suggests an association between clinical and subclinical hypothyroidism, and type 2 diabetes mellitus, but many of these studies have small sample sizes, and the literature includes a lot of inconclusive data [4].

This could be explained by the fact that thyroid hormones are necessary for all metabolic reaction sin the body. With better thyroid functioning, we have better insulin secretion, less glucose absorption from the gut and kidneys, more incretin GLP1, and morning dopamine surge, more glucose uptake in muscles, less inflammation, better colon microbiota, less hepatic glucose production and less lipolysis, when all impaired, they lead to worse diabetes status.

Although both groups had significantly lower TSH results after hypothyroidism treatment, a statistically significant reduction of median HbA1C was only observed in women (Table 3). This finding should be subject to further investigation in order to check whether it is due to a sampling bias (because our study was monocentric and included only the patients who followed up in this center) or if it's a common finding in the whole Lebanese population. However, Elderly women were a special group in which the association between poor glycemic control and the risk of subclinical hypothyroidism was higher [17]. 
Monocentric Retrospective Study Assessing the Association Between Clinical and Subclinical Hypothyroidism and Type 2 Diabetes Mellitus (Type 2 DM) and the Benefit of Treating Hypothyroidism on the Improvement of Diabetes Control

Our results suggest that patients who had no comorbidities were the ones who took the most advantage of the hypothyroidism treatment, so they had significantly lower HbA1C after hypothyroidism treatment, compared to the patients who had one or more comorbidities. On one hand, diabetes itself can cause these comorbidities, knowing that the majority of our sample's comorbidities were cardiovascular, and that diabetes could damage the cardiovascular system. On the other hand, a higher prevalence of cerebrovascular diseases was noted among type 2 diabetes mellitus patients with hypothyroidism compared to those with euthyroidism after adjustment for age and gender [10]. Furthermore, a meta-analysis suggested that subclinical hypothyroidism may be associated with the increase of diabetic complications, specifically diabetic nephropathy ( $\mathrm{OR}=1.74)$, diabetic retinopathy ( $\mathrm{OR}=1.42$ ), peripheral arterial disease (OR $=1.85)$, and peripheral neuropathy (OR = 1.87) [4]. This finding was replicated in many other studies that followed [7-9]. Further studies are needed in this context to clarify the causality in this relationship between hypothyroidism treatment, lower HbA1C levels, and the presence of diabetes complications.

Finally, since thyroxine replacement therapy has protective effects on the renal function and the cardiovascular system as well [18], and patients who had thyroid dysfunction among diabetics were those who had a longer diabetes duration, our results suggest a clinical utility of screening type 2 diabetic patients for hypothyroidism and treating it accordingly. Moreover, evidence supports the necessity to screen thyroid function in patients with type 2 diabetes [11,12], and appropriate treatments should be given accordingly as well [4]. Furthermore, although there's a need for randomized trials to verify this finding, thyroid hormone replacement therapy might probably decrease the risk of cardiovascular diseases in patients with diabetic nephropathy [13]. A prospective study including 75,076 American women aged 20-89 years followed during a median of 28 years found out that hypothyroid women had increased mortality risks for diabetes mellitus, cardiovascular disease, and cerebrovascular disease [19]. However, further economic studies must be done in order to prove the feasibility and economical interest of such a screening, as well as its frequency and interest in terms of complications prevention.

\section{Conclusion}

In conclusion, this study assessed the prevalence of hypothyroidism among diabetic patients in Lebanon. It also proved the utility of treating hypothyroidism on HbA1C levels, especially for patients who didn't have any other comorbidity. Besides, we found that this study showed that the patients who received hypothy- roidism treatment had significantly lower HbA1C levels (p-value $=0.001$ ). Yet, statistically significant. Further research is needed to assess the economic benefit of screening all diabetic patients for hypothyroidism and treating them.

\section{Bibliography}

1. American Diabetes Association. "Diagnosis and Classification of Diabetes Mellitus". Diabetes Care 32 (2009): S62-67.

2. Ogurtsova K., et al. "IDF Diabetes Atlas: Global estimates for the prevalence of diabetes for 2015 and 2040". Diabetes Research and Clinical Practice 128 (2017): 40-50.

3. IDF. "Diabetes prevalence in Lebanon" (2017).

4. Han C., et al. "Subclinical Hypothyroidism and Type 2 Diabetes: A Systematic Review and Meta-Analysis". PloS One 10.8 (2015): e0135233.

5. Al-Geffari M., et al. "Risk Factors for Thyroid Dysfunction among Type 2 Diabetic Patients in a Highly Diabetes Mellitus Prevalent Society". International Journal of Endocrinology 2013 (2013): 417920.

6. Wang C. "The Relationship between Type 2 Diabetes Mellitus and Related Thyroid Diseases". Journal of Diabetes Research 2013 (2013): 390534.

7. Zhao W., et al. "A high thyroid stimulating hormone level is associated with diabetic peripheral neuropathy in type 2 diabetes patients". Diabetes Research and Clinical Practice 115 (2016): 122-129.

8. Zhou J-B., et al. "Subclinical hypothyroidism and the risk of chronic kidney disease in T2D subjects: A case-control and dose-response analysis". Medicine (Baltimore) 96.15 (2016): e6519.

9. Mansournia N., et al. "Subclinical hypothyroidism and diabetic nephropathy in Iranian patients with type 2 diabetes". Journal of Endocrinological Investigation 40.3 (2017): 289-295.

10. Song F., et al. "The prevalence and determinants of hypothyroidism in hospitalized patients with type 2 diabetes mellitus". Endocrine 55.1 (2017): 179-185.

11. Hollowell JG., et al. "Serum TSH, T (4), and thyroid antibodies in the United States population (1988 to 1994): National Health and Nutrition Examination Survey (NHANES III)". The Journal of Clinical Endocrinology and Metabolism 87.2 (2002): 489-499. 
12. Perros P., et al. "Frequency of thyroid dysfunction in diabetic patients: value of annual screening". Definition, Diagnosis and Classification of Diabetes Mellitus 12.7 (1995): 622-627.

13. Seo C., et al. "Thyroid Hormone Replacement Reduces The Risk Of Cardiovascular Diseases In Diabetic Nephropathy Patients With Subclinical Hypothyroidism". Endocrine Practice 4.3 (2018): 265-272.

14. CDC. Principles of Epidemiology | Lesson 3 - Section 2 (2017).

15. Centeno Maxzud M., et al. "Prevalence of thyroid dysfunction in patients with type 2 diabetes mellitus". Medicina (Mex) 76.6 (2016): 355-358.

16. Dimic D., et al. "The effect of metformin on TSH levels in euthyroid and hypothyroid newly diagnosed diabetes mellitus type 2 patients". Bratislavske Lekarske Listy 117.8 (2016): 433-435.

17. Cho JH., et al. "Poor glycemic control is associated with the risk of subclinical hypothyroidism in patients with type 2 diabetes mellitus". Korean Journal of Internal Medicine 31.4 (2016): 703711.

18. Aziz KMA. Association of Hypothyroidism with Body Mass Index. "Systolic Blood Pressure and Proteinuria in Diabetic Patients: Does treated Hypothyroidism with Thyroxine Replacement Therapy Prevent Nephropathy/Chronic Renal Disease?" Current Diabetes Reviews 12.3 (2016): 297-306.

19. Journy NMY., et al. "Hyperthyroidism, Hypothyroidism, and Cause-Specific Mortality in a Large Cohort of Women". Thyroid Journal Archives / American Thyroid Association 27.8 (2017): 1001-1010.

\section{Assets from publication with us}

- Prompt Acknowledgement after receiving the article

- Thorough Double blinded peer review

- Rapid Publication

- Issue of Publication Certificate

- High visibility of your Published work

Website: www.actascientific.com/

Submit Article: www.actascientific.com/submission.php

Email us: editor@actascientific.com

Contact us: +919182824667

Citation: Mohammad Rida Farhat, et al. "Monocentric Retrospective Study Assessing the Association Between Clinical and Subclinical Hypothyroidism and Type 2 Diabetes Mellitus (Type 2 DM) and the Benefit of Treating Hypothyroidism on the Improvement of Diabetes Control". Acta Scientific Nutritional Health 5.5 (2021): 03-09. 